\title{
Integrating QoS Restrictions into the Process of Service Selection
}

\author{
Claudia Linnhoff-Popien and Dirk Thißen
}

Aachen University of Technology, Department of Computer

Science IV, Ahornstr. 55, D-52056 Aachen, GERMANY

E-mail: \{popien, thissen\}@i4.informatik.rwth-aachen.de

Phone: +49-241-8021415, Fax: +49-241-8888220

\begin{abstract}
The growing of computer networks and transmission capacity causes the possibility of a rising amount of service offers in distributed systems. One of the CORBAservices is the trading service, which supports clients in searching for suitable services.

This paper introduces an evaluation component for a CORBA trader. This component uses a service distance function to select an optimal service offer from a trader's database. A service distance function computes the distance between a service request and a service offer with regard to their service properties. The service with the minimal distance to the service request is the optimal service. Hence, existing methods for distance computation between vectors are used, and a rulework for computing the distance between service properties is developed. The resulting evaluation procedure is implemented in a CORBA trader using the distributed platform Orbix. The implementation is compared with the classic evaluation mechanism of the used trader. Furthermore the various methods for distance computation are compared among themselves.
\end{abstract}

\section{Keywords}

CORBA; Trading service; Quality of service; Service distance.

\section{INTRODUCTION}

In a large distributed system many services are offered. To support a client in searching a special service a trader can be used. This trader has a service directory, which contains available services specified by a service type and service property values. If a client requests a service, the trader procures a convenient one. The 
problem in this mechanism is that the process of selecting a service only matches the client's request against the service property values. There is no possibility to take into consideration quality of service aspects as defined in the quality of service basic framework [QoS 95]. Hence it follows, that two problems in the common trading mechanism arise:

1. There is no possibility to identify an order of precedence on the fitting service offers. So the trader is able to procure a minimal suitable service whereas better services are registered in the trader's service directory.

2. If all service offers in the trader's domain have a small deviation from the importer's specification, no service is procured. Nevertheless a little deviating service could satisfy the importer.

This paper treats a modification of a trader's evaluation procedure. The modification allows the client to include quality aspects into its request. The trader now uses a set of service distance functions to calculate the distance between the service request and each service offer to investigate a ranking on the given service offers. Therefore, a service is viewed as a vector of properties. Analytic methods are used to calculate distances between such vectors. With respect to service quality a property can consist of several values with certain roles. Thus, to calculate a distance between these components, it was necessary to develop a new rulework. The service with minimal distance to the requested service is regarded as optimal service.

The paper is structured as follows. In the second chapter an overview about service trading and quality effects is given. The trading process and service notations are introduced. Furthermore the consideration of service quality leads to the concept of the service distance functions. The third chapter explains the concept of finding an optimal service in a trader's service directory. An overview about the implementation of the presented evaluation approach into a CORBA trader is given in the fourth chapter. Chapter five presents as well a comparison between the new evaluation component and the existing mechanism as a comparison between the used distance computation methods. Finally, the sixth chapter presents conclusions and tasks for further studies.

\section{SERVICE REQUEST VS SERVICE OFFER}

To explain the purpose of service distance functions, at first a short introduction into service notations and service trading is given.

\subsection{Service trading in CORBA}

A service is a function provided by an object at a computational interface [PSW 96]. This function expresses a set of capabilities available at the interface. Such a service is an instance of a service type. For each service type there exists an affiliated interface type and several noncomputational aspects called service properties. Different services of the same service type may differ in their service proper- 
ties. A service property is described as a (name, value) pair and is an instance of a service property type.

A trader is a service procuring other services. For this purpose, a trader needs a service directory, which contains descriptions about all services available at the trader. A service description included in such a directory is called a service offer.

An exporter, that is a server that wants to provide a service, can register this service within the service directory. The service is registered with exporter identifier, service interface identifier, service type description and service properties.

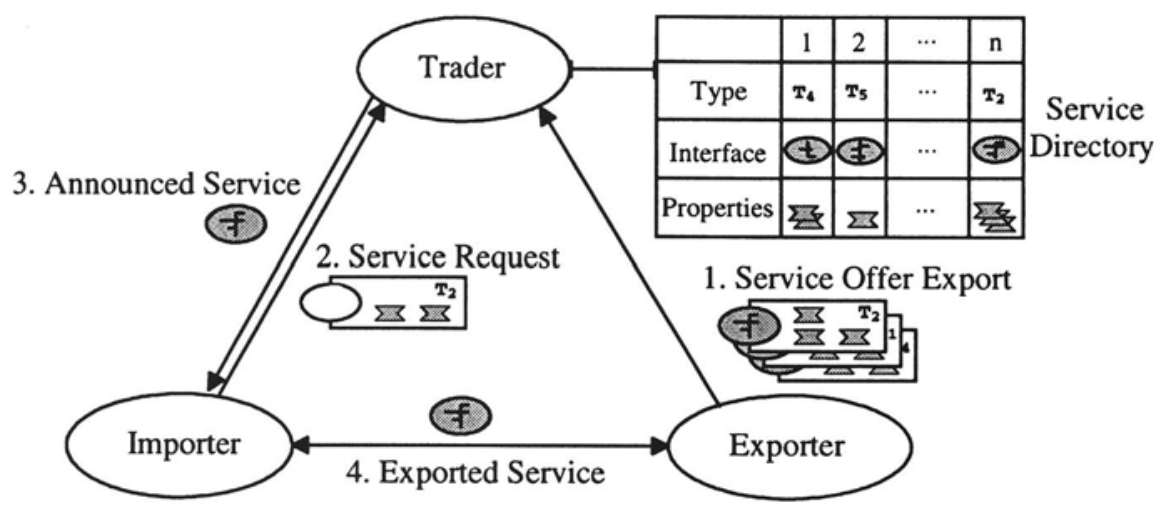

Figure 1 Service Trading

A client using a trader to search a service is called importer [SPM 94]. It requests a service at the trader in specifying the service type and service properties. Therefore the importer chooses the operations SEARCH or SELECT. SEARCH brings about all suitable services, whereas SELECT brings about only one of them. Such specifications for example could be made by a service description language [PM 94]. The choice of suitable services at the trader is made by matching service type and service properties with every service offer. The result of a service import is an interface identifier. Now, the importer can directly contact the server that has exported the service. This trading process is shown in figure 1.

\subsection{Quality of service and service properties}

The described trading mechanism only tolerates single values for each service property. These values are matched against values of the service offer. A single demanded value is either fulfilled or not. This concept is insufficient for many applications. Many services like video conferencing and interactive applications need a consideration of quality aspects. The quality of service could be taken into account by formulating quality characteristics as service properties. Quality characteristics are e.g. throughput, transmission delay or availability [Th 95]. Now, an importer needs to specify more than one value, namely a target, a lower bound and an upper bound on such a characteristic to formulate its wishes and limits, see 
figure 2. All these values are components of a quality of service parameter, so they all are needed to express quality [QoS 95]. Furthermore the importer must be allowed to specify the preference of every characteristic, so that the trader knows what characteristics are to be dealt with priority. Because of the arising complex structures of service properties, normal matching no longer can be used.

\section{Service Properties}

Service Request: $\mathbf{r}=\left(r_{1}, \ldots, r_{n}\right)$ with Service Offer $\mathbf{o}=\left(o_{1}, \ldots, o_{m}\right)$ with

Service Request Property $r_{i}$ :

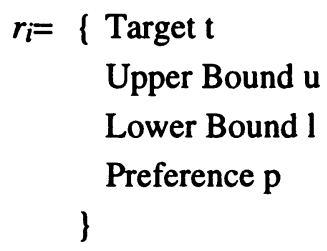

Service Offer Property $o_{i}$ :

$$
o_{i}=\left\{\begin{array}{l}
\text { Upper Bound u } \\
\text { Lower Bound } 1
\end{array}\right.
$$

Figure 2 Service Property Structure

A number of distance functions on vectors are well known in analysis, especially maximum metric, Euclidean metric and Manhattan metric. Furthermore methods in fuzzy set theory and the analytic hierarchy process provide approaches suitable for the distance computing on vectors. But there is no method to compute the distance between single components, because they are records of values with different roles. So, it was necessary to develop a distance function on service property records. This distance function is presented in the next chapter, just as a short overview of known distance functions on vectors.

\section{SERVICE DISTANCE FUNCTION}

To compute the distance between service vectors, two steps are necessary. The first step is to compute the distance between the components of both vectors, the service property records. This step is described in section 3.1. The result is a vector of differences. In the second step, these differences must be combined to the distance between vectors. In section 3.2 some methods are presented to achieve this. Section 3.3 presents a short comparison of the presented service distance functions.

\subsection{Service property record distance}

As described in chapter two, a service request property with quality consideration in our example consists of four values with the roles target, upper bound, lower bound and preference. A service offer property consists of a lower bound and an upper bound. So, it is impossible to compute a simple difference between both structures. Therefore, it was necessary to draw up rules for every possible combination of values. 
The importer is allowed to specify each combination of target and bounds. To simplify the evaluation, in the specifying process either both or no bounds are occupied. Not occupied components have the value undefined. Likewise the exporter can specify only one or both bounds. Service offer vector and service request vector need not have equal size and contain equal properties, so $o_{i}$ only means the property with the same name as $r_{i}$. In this paper only a short insight into the rulework is given. The complete rulework is explained in [Th 96].

- If the service request property $r_{i}$ only defines a target, the difference is zero if the value is within the bounds of the affiliated service offer property $o_{i}$. Outside the bounds the difference to the corresponding bound must be computed:

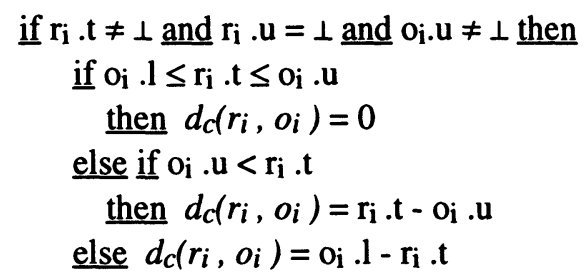

The rules for the cases that only bounds or bounds and a target are specified, are treated similar. Furthermore there are other problems that must be considered, e.g. properties could be specified in a non-numerical format or in a probabilistic way.

At last, the resulted difference must be weighted with the importer specified weights to consider the importance of a property. The result is a weighted component distance $d_{w c}$ :

$$
d_{w c}\left(r_{i}, o_{i}\right)=r_{i} \cdot p * d_{c}\left(r_{i}, o_{i}\right)
$$

The whole set of rules allows to compute the distances in all components of the service request vector. The resulting differences could be put together in a difference vector $\mathbf{d}=\left(d_{1}, \ldots, d_{n}\right)$ with $d_{i}=d_{w c}\left(r_{i}, o_{i}\right)$ for $\mathrm{i}=1, \ldots, \mathrm{n}$. In the next step all these values $d_{i}$ are to be combined to the difference between the service vectors.

\subsection{Service property vector distance}

The idea of a vector distance function $d_{v}$ is to compute a distance between vectors by combining the individual component differences. In analysis there are a few metrics which can be employed on such vectors. In general, an analytic metric for $\mathrm{n}>0$ is defined as follows:

$$
d_{v}\left(\left(x_{1}, \ldots, x_{m}\right),\left(y_{1}, \ldots, y_{m}\right)\right):=\sqrt[n]{\sum_{i=1}^{m} d_{w c}\left(x_{i}, y_{i}\right)^{n}}
$$

In practice, the relevant cases are $n=1,2$ and infinity. In these cases, the metrics are called Manhattan metric, Euclidean metric and maximum metric. The optimal service offer can be found by minimising the distance $d_{v}$. Beside the mathematical functions to compute the distance between vectors, in practice there are other methods to find out an optimal service: 
- Fuzzy set theory [Zi 91]

- Analytic hierarchy process (AHP) [Sa 80], [DP 94]

- Additive and multiplicative model [Be 90]

A description of these methods can be found in [Th 96].

\subsection{Comparison}

In the previous sections service distance functions $d_{w c}$ and $d_{v}$ were presented. There is no other function to compare with $d_{w c}$, because the property records have a new structure. So the service ability of $d_{w c}$ is to be examined in practice. For $d_{v}$, several methods are presented. The additive model needs not to be considered, because it is equal to Manhattan metric. Table 1 gives a short overview about computation complexity, expected result precision and implementation easiness for all vector difference computing methods.

Table 1 Overview about the presented methods

\begin{tabular}{lcccc}
\hline & metrics & fuzzy set theory & AHP & multiplicative model \\
\hline complexity & + & + & - & $\circ$ \\
precision & + & + & ++ & + \\
implementation & + & + & - & + \\
\hline
\end{tabular}

Because of the easy implementation possibilities of all methods except the analytic hierarchy process, the best solution is an integrated distance computation mechanism with choice of the distance function by the importer.

\section{IMPLEMENTATION OF THE EVALUATION COMPONENT}

In this chapter, concepts are presented for implementing the trader evaluation component in $\mathrm{C}++$. The evaluation component is integrated in an existing CORBA trader [Zl 96] of the distributed platform Orbix [ORBIX]. Different importers could have different meanings about an optimal service. Therefore, in the evaluation component every combination of a distance function with both, multiplicative and exponential weightings is possible.

The importer must specify the service request as before, only additionally it can specify more values on a service property. Furthermore, the importer must choose a service distance function and a weighting mechanism. Hence, two types are defined, distance_type and preference_type. The first type includes the methods for computing a service property vector distance as presented in chapter 3.2 , the second type contains a set of weighting mechanisms. Fuzzy set theory optimisation and the additive model can be viewed as special cases of maximum met- 
ric respectively Manhattan metric. Because every combination of distance functions with preference functions is allowed, these types do not need to be considered. To be consistent with the trading standard, the service properties can not be transmitted to the trader as vector, but must be transformed in policies. Within the trader, this transformation must be annulated.

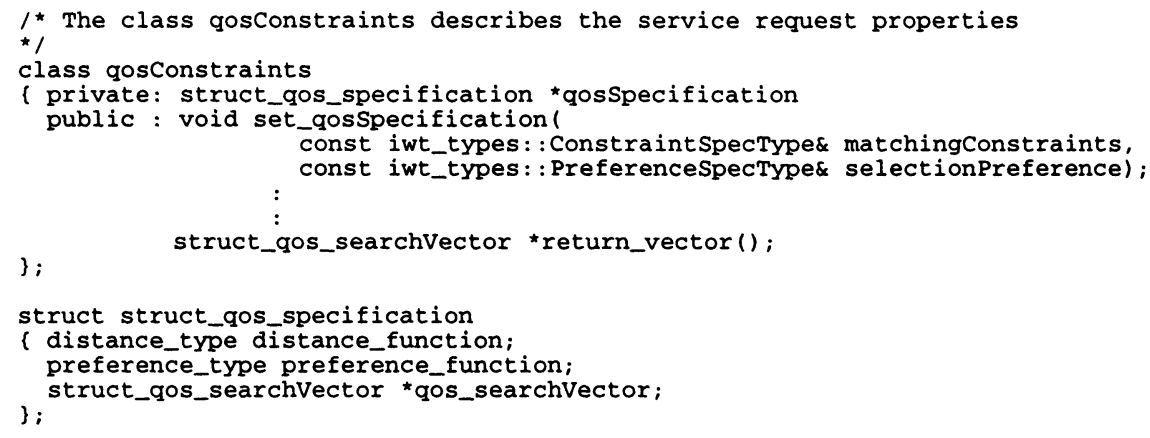

Figure 3 Class qosConstraints for a service request

Because Orbix and the used trader are object oriented, the quality of a service request, i.e. the service properties, are seen as an object of the class qosconstraints, see figure 3 . The internal state qosspecification is a vector containing the desired distance and preference function as well as a vector of struct_qos_searchVector. For each service request property, this vector includes a record of target, upper bound, lower bound and preference. The public function set_qosSpecification serves for covering the qosSpecification with the specified matching constraints. The specification is contained in the variable matchingConstraints. selectionPreference contains the preferences on the properties specified in matchingConstraints. Both are defined in the existing trader. Furthermore, functions must be implemented to get the registered qosSpecification. More work is not needed on an object for a service request vector.

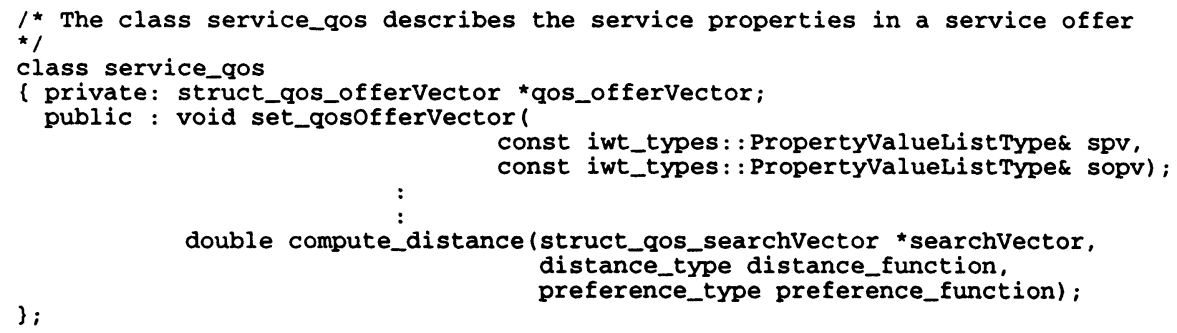

Figure 4 Class service_qos for a service offer

Likewise the property specification is seen as an object. For service request and service offer different objects are necessary, because a service provider can specify 
fewer values on a property. So, the internal state qos_offerVector of an object for service offer properties is a vector which contains a record consisting of upper and lower bound for each service offer property. A function set_qos_offervector is needed to cover the vector with the exporter's service property and service offer property values. Then, the distance to a service request specification is to be computed using the function compute_distance. This function realises the distance functions described in chapter three. The class service_qos for the service offer properties is shown in figure 4.

The described objects are implemented and integrated into the CORBA trader. In the trader, all service offers are stored into a service directory as objects of class ServiceTableItem. Such an object generally contains a property value list. Instead of this, at the service export now an object service_qos for each service is generated. At a service import, the presented matching constraints are transformed into an object gosConstraints. This object is passed to the service directory, and then to the service_qos of each ServiceTableItem. Within this object, the service distance between the service request and the affiliated service offer is computed. All suitable service offers are collected in a list which is sorted by the computed distance. So, the first entry is the optimal service.

\section{ASSESSMENT OF THE EVALUATION COMPONENT}

To assess the evaluation component, a comparison with the classic trader will be necessary. Furthermore, the different distance functions should be compared with each other. For this purpose, two comparisons are made. First, the evaluation times of all methods are compared. Subsequently, a comparison of the import results is given.



Figure 5 The measure points

To investigate the trader's evaluation time, four measure points have been established. These points are shown in figure 5. Nothing happens between $A$ and $B$ in the classic trader. In the new trader the transformation of the matching constraints into a service request property vector is made there. The evaluation process takes place between $B$ and $C$. Between $C$ and $D$ only a copy of the resulted service offer is made in both traders. The only exception is the AHP. At this method, pairwise comparisons between all service offers are necessary. Therefore, after passing 
point $C$ the matrices for pairwise comparison must be evaluated. To do so, the time spans $A B, B C$ and $C D$ are measured.

In the following, all service offers have the same service type and the same service properties. The service property values are chosen by chance. Furthermore the service request has the same service type and a subset of the properties of the service offers.

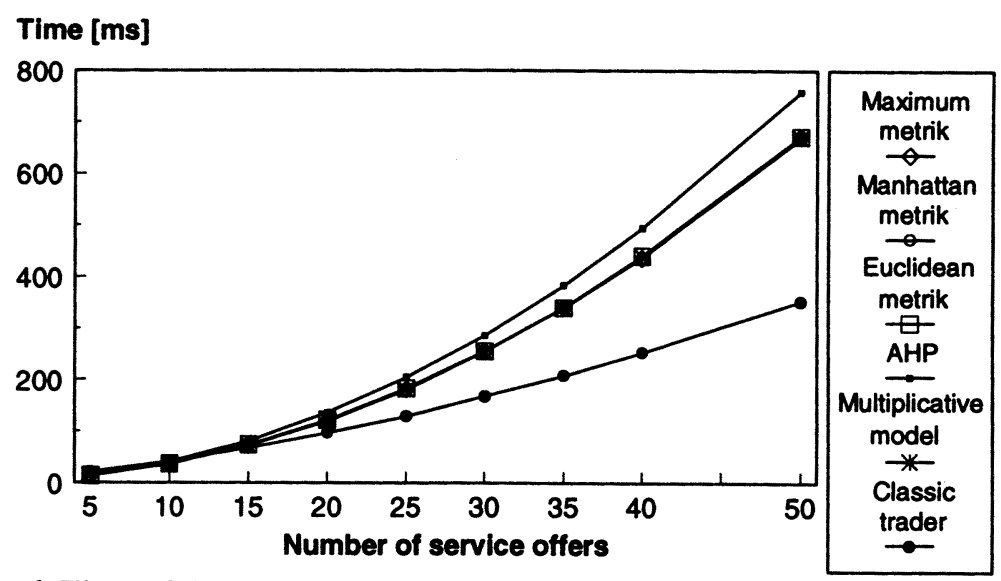

Figure 6 Time of the overall evaluation process

Figure 6 shows the overall evaluation time the trader needs depending on the number of service offers with correct service type in the trader's database, i.e. $A D$. The service request is made with five of the service offers' properties. All evaluation mechanisms show an exponential ascent in the evaluation time. The reason is the implementation of sequences in Orbix. All suitable services are collected in a sequence of the type serviceofferDetailType. If a suitable service is found, a new sequence with an increased length is generated. Then, the content of the old sequence must be copied into the new one. With an increasing number of services, the information which has to be copied increases as well, resulting in an exponential ascend of evaluation time. On the same reason the classic trader is faster than the new one, because there is a lower amount of information that must be copied.

The behaviour of the AHP is slightly different from that of the other distance functions. To explain that phenomenon it is necessary to split the evaluation time into the times $A C$ and $C D$. $A B$ needs not to be considered individual, as this is only one millisecond on average. Figure 7 shows the time between $C$ and $D$. At the use of the AHP at $C D$ the pairwise comparison takes place. For each service request property a matrix is generated. For each new service offer, the size of all matrices increases, resulting in an exponential ascend. The other distance functions and the classic trader only make a copy of the resulted service offers; in these cases the time increases constantly. 


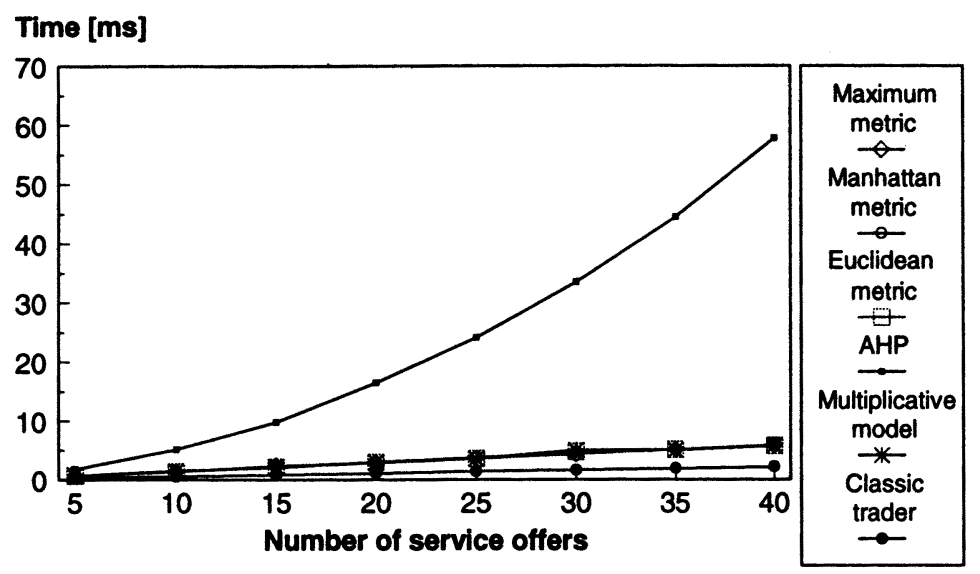

Figure 7 Time after the search in the service directory

Time behaviour of the classic trader is more suitable than the new one's, but this results by the implementation of the Orbix sequences. By implementing own sequences, this disadvantage becomes lost.

Now the result of the service import should be examined. Ten service offers with the correct service type and ten service properties are exported. These services are imported and the distance to each service is considered. The sum of the distances is standardised to one, to make possible a comparison between the distance functions.

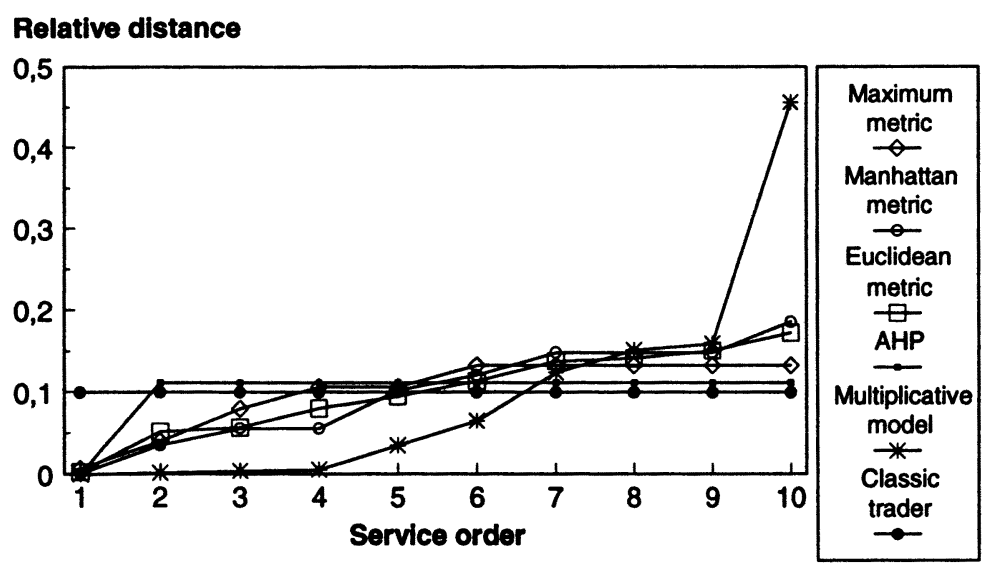

Figure 8 Distances to all imported services

Figure 8 shows the distance to each service offer for all distance functions and the classic trader by specifying a service request with ten properties. The figure shows a similar behaviour of the metrics. The multiplicative model shows a more extreme behaviour. The more suitable services are very well evaluated and with only small differences between them. That results from the fact that large differ- 
ences in service properties are weighted stronger than low differences. The less suitable services are evaluated very disadvantageous and with large differences. The AHP evaluates the best service offer very good, the other service offers are valued very bad. The reason is the comparison strategy used. The classic trader values all services equally, the first service found is selected. It should be mentioned that the service order resulting from the use of a distance function is depending on that function.

The behaviour of the distance functions leads to the idea of extending the operators SEARCH and SELECT. The importer could specify a distance of the interval $[0,1]$. Only those service offers with a relative distance lower than the importer's distance parameter are presented. Such a variation of the importer's operations is not possible with the classic trader.

Altogether the new evaluation component has an advantage compared to the classic trader. But in further investigations the suitability of the analytic hierarchy process appeared as very low. Therefore the pairwise comparison of the analytic hierarchy process should be revised.

\section{CONCLUSIONS}

This paper has presented an approach to formulate and evaluate service properties with respect to quality. For that purpose services were viewed as vectors, so it was possible to compute the distances between a service request and each service offer. An optimal service offer has a minimal distance to the service request. Furthermore, aspects are presented to implement the service distance computing method in an Orbix trader. The implementation was tested and the new evaluation mechanism was compared with the classic trader. Additional, a comparison between the distance functions was given.

The result of this paper is an evaluation mechanism for distance computing between services. This rulework is more flexible and more importer oriented than common selection mechanisms, because it allows the importer on the one hand to specify the quality of its properties. On the other hand it allows to express its interpretation of an optimal service. So the importer gets better services. Because the disadvantage of distance functions in evaluation time is caused by the implementation of Orbix, the evaluation component altogether is more suitable than the old mechanism. Furthermore an extended importer operation SEARCH or SELECT should be implemented using service distance functions.

The future tasks are to enhance the rulework by considering threshold on service properties and enabling the description of all service property values as probabilistic or statistic. Another point of interest is the distinction between static and dynamic properties. Further work has to be done on optimising the trader's time behaviour, e.g. by transforming non-numerical service properties into a numerical format at the importer. Furthermore it is necessary to modify the pairwise comparison in the analytic hierarchy process. Otherwise this method is useless. 


\section{REFERENCES}

[Be 90] Bernard, R.: Decision-Aid and Decision-Making. In: Bana e Costa, C.: Readings in Multiple Criteria Decision Aid; Springer-Verlag, 1990.

[DP 94] Douligeris, C.; Pereira, I.: A Telecommunications Quality Study Using the Analytic Hierarchy Process. IEEE Journal on Selected Areas in Communications, Vol. 12, No. 2, pp. 241-50, Feb. 1994.

[ORBIX] Orbix - programmers guide \& reference manual. IONA Technologies Ltd., Release 2.0, 1996.

[PM 94] Popien, C.; Meyer, B.: A Service Request Description Language. In: Hogrefe, D; Leue, S.: Formal Description Techniques VII; Chapman \& Hall, 1994.

[PSW 96] Popien, C.; Schürmann, G.; Weiß, K.-H.: Distributed Processing in Open Systems (in German). Teubner, 1996.

[QoS 95] ISO/IEC JTC1/SC21/N9309: Open System Interconnection, data management and Open Distributed Processing - Quality of Service, Basic Framework - Working Draft, January, 1995.

[Sa 80] Saaty, T.L.: The Analytic Hierarchy Process. McGraw-Hill, 1980.

[SPM 94] Spaniol, O.; Popien, C.; Meyer, B.: Services and Service Trading in Client/Server Systems (in German). Thomson Publishing GmBH, 1994.

[Th 95 ] Thißen, D.: New Concepts of QoS (in German). Thesis at the Department of Computer Science IV at Aachen University of Technology, April 1995.

[Th 96] Thißen, D.: QoS based Optimisation of Service Selection within an Orbix Trader (in German). Diploma thesis at the Department of Computer Science IV at Aachen University of Technology, July 1996.

[Zi 91] Zimmermann, H.-J.: Fuzzy Set Theory and its Applications, 2nd edition. Kluwer Academic Publishers, 1991.

[Zl 96] Zlatintsis, S.: Design and Valuation of a Trader Gateway between ANSAware and ORB systems (in German). Diploma thesis at the Department of Computer Science IV at Aachen University of Technology, February 1996.

\section{BIOGRAPHY}

Claudia Linnhoff-Popien has studied mathematics and theoretical computer science in Leipzig Germany, Diploma 1989. After a research work at Technical University of Magdeburg she became an assistant at Aachen University of Technology in the Department of Computer Science in 1991. She finished her Ph.D. thesis in 1994. At the present, she works on her habilitation thesis at Aachen University of Technology.

Dirk Thißen has studied computer science at Aachen University of Technology in Germany. In 1997 he got his diploma. Then he became a research assistant at the Department of Computer Science at Aachen University of Technology. 